\title{
FERTIRRIGAÇÃO DA BANANEIRAcv. PRATA-ANÃ COM N E K EM UM ARGISSOLO VERMELHO-AMARELO ${ }^{1}$
}

\author{
VALBÉRIO PAOLILO DOS SANTOS², PEDRO DANTAS FERNANDES ${ }^{3}$, ALBERTO SOARES DE MELO ${ }^{4}$, \\ LAFAYETTE FRANCO SOBRAL ${ }^{5}$, MARCOS ERIC BARBOSABRITO ${ }^{6}$, \\ JOLLY DAYANNE DE MELO DANTAS ${ }^{6}$, LILIANNI VIEIRABONFIM ${ }^{6}$
}

RESUMO-O Nordeste brasileiro é a principal região produtora de banana, responsável por cerca de 35,80\% da produção nacional. Apesar das condições favoráveis ao seu cultivo, a produtividade tem sido muito abaixo do seu potencial. Geralmente, isso se deve a problemas de suprimento hídrico e nutricional. Dentre os nutrientes, o nitrogênio $(\mathrm{N})$ e o potássio $(\mathrm{K})$ são requeridos em maiores quantidades pela bananeira. Com o objetivo de estudar o efeito de quatro doses de N (0; 117; 235 e $352 \mathrm{~kg} \mathrm{ha}^{-1}$ de N) e de K (0; 157; 313 e $470 \mathrm{~kg}$ $\mathrm{ha}^{-1} \mathrm{de}_{2} \mathrm{O}$ ), aplicados via fertirrigação, avaliaram-se características produtivas de um $2^{\circ}$ ciclo de cultivo da banana cv. Prata-Anã, em um Argissolo Vermelho-Amarelo dos tabuleiros costeiros do Nordeste brasileiro. O N não influenciou na produção de banana, enquanto a resposta ao K foi linear. O nível crítico de K pelo Mehlich-1, na profundidade de 0,20- 0,40 m, foi 0,71 mmol $\mathrm{kg}^{-1}$.

Termos para indexação: Irrigação, adubação, nutrição.

\section{FERTIGATION OF BANANA CV PRATAANÃ WITH N AND K IN AN ULTISOL}

ABSTRACT-The Brazilian Northeast is the main producing area, being responsible for about 35,80\% of banana's national production. In spite of the favorable conditions to its cultivation, the average national yield has been a lot below its potential, due mainly to water and nutrients supply. Among all nutrients, nitrogen $(\mathrm{N})$ and potassium $(\mathrm{K})$ are the ones required in larger amounts by the banana. With the objective of studying the effect of four rates of $\mathrm{N}\left(0,117,235\right.$ and $352 \mathrm{~kg} \mathrm{ha}^{-1}$ of $\left.\mathrm{N}\right)$, and of four rates $\mathrm{K}(0,157,313$ and 470 $\mathrm{kg} \mathrm{ha}^{-1}$ of $\mathrm{K}_{2} \mathrm{O}$ ) applied by fertirrigation in the $2^{\circ}$ cycle of cultivation of the cv. Prata Anã, data was collected in an factorial experiment in randomized blocks, in an Ultisol of the coastal tableland, of the northeast of Brazil. There was not response to $\mathrm{N}$ but a linear one was observed for K. Soil K by Mehlich-1 critical level at $0,20-0,40 \mathrm{~m}$ was $0,71 \mathrm{mmol}_{\mathrm{c}} \mathrm{kg}^{-1}$.

Index Terms: Irrigation, fertilization, nutrition.

\section{INTRODUÇÃO}

A produção de banana no Brasil ultrapassou 6,5 milhões de toneladas em 2003. O Nordeste brasileiro é a principal região produtora, sendo responsável por cerca de 35,80\% da produção nacional. Apesar das condições favoráveis ao seu cultivo, a produtividade tem sido muito abaixo do seu potencial em função de problemas de suprimento hídrico e nutricional.

Dentre os nutrientes, o $\mathrm{N}$ e o $\mathrm{K}$ são requeridos em maiores quantidades pela bananeira. O primeiro é muito importante para o crescimento vegetativo da planta, principalmente nos três primeiros meses, quando o meristema está em desenvolvimento (Warner \& Fox, 1977). Também é responsável pelo aumento no número de pencas, emissão e crescimento dos rebentos, aumentando consideravelmente a quantidade total de matéria seca (Lahav \& Turner, 1983). O segundo é importante não só na translocação de fotoassimilados e no balanço hídrico, mas também na produção da bananeira, melhorando a qualidade dos frutos e sua resistência (Borges \& Oliveira, 2000).

A adubação de forma tradicional, nos cultivos irrigados, vem sendo substituída pela aplicação de fertilizantes via água de irrigação. A fertirrigação, além de ser de grande utilidade para as plantas, pois o nutriente é fornecido juntamente com a água (essencial para sua absorção), apresenta ainda muitas

${ }^{1}$ (Trabalho 227-07). Recebido em: 25-09-2007. Aceito para publicação em: 27-04-2009.

${ }^{2}$ Eng. Agrônomo, EMDAGRO - Empresa de Desenvolvimento Agropecuário de Sergipe, Aracaju -SE. E-mail: valberio.santos@emdagro.se.gov.br

${ }^{3}$ Professor Titular, Eng. Agrícola, UFCG, Campina Grande-PB. E-mail: pdanats@deag.ufcg.edu.br

${ }^{4}$ Professor Titular, Ciências Agrárias, UEPB, Catolé do Rocha-PB. E-mail: alberto@uepb.edu.br

${ }^{5}$ Pesquisador, Embrapa Tabuleiros Costeiros, Aracaju-SE. E-mail: lafayette@cptac.embrapa.br

${ }^{6}$ Estudante de Engenharia Agronômica, bolsista PIBIC-CNPq, UFS, São Cristóvão -SE 
outras vantagens, entre as quais a de melhor distribuição do fertilizante no campo e a possibilidade de maior parcelamento das adubações, aumentando a eficiência na utilização dos adubos pelas plantas (Duenhas et al., 2002).

Guerra et al. (2004) avaliaram o efeito da frequência da fertirrigação com N e K em relação à adubação convencional, na produção e qualidade de frutos da bananeira Prata-Anã e observaram que a maior frequência promoveu valores significativamente maiores na produção de banana em comparação com a adubação convencional. Sousa et al. (2004) avaliaram o efeito do N e do K, aplicados via água de irrigação por microaspersão sobre as características de produção da bananeira Grand Naine, no segundo ciclo, em um Argissolo, e não observaram efeito da aplicação de $\mathrm{N}$ na produção de banana, enquanto o $\mathrm{K}$ aumentou a produção, atingindo valor máximo, com a aplicação de 933,33 $\mathrm{kg} \mathrm{ha}^{-1}$ de $\mathrm{K}_{2} \mathrm{O}$.

Teixeira et al. (2001) observaram reduções significativas do teor de $\mathrm{K}^{+}$no solo, nas profundidades de 0 a $0,20 \mathrm{~m}$ e de 0,20 a $0,40 \mathrm{~m}$, em dois ciclos de produção da bananeira Nanicão sob irrigação, e indicaram que a depleção desse nutriente disponível pode comprometer a produção, considerando as grandes quantidades do nutriente requeridas pela bananeira.

O presente trabalho teve como objetivo determinar as melhores combinações de doses de $\mathrm{N}$ e de $\mathrm{K}$, aplicadas via fertirrigação, no $2^{\circ}$ ciclo da cultura da banana cv. Prata- Anã, em Argissolo Vermelho-Amarelo dos tabuleiros costeiros, associadas às características produtivas da planta e ao nível crítico de K no solo.

\section{MATERIAL E MÉTODOS}

O estudo foi realizado na Estação Experimental Câmpus Rural do Departamento de Engenharia Agronômica, da Universidade Federal de Sergipe - UFS, localizado na porção central da região fisiográfica do Litoral do Estado de Sergipe, a 15 km de Aracaju, nas coordenadas geográficas de $10^{\circ} 55^{\prime}$ $27^{\prime \prime}$ de latitude sul e $37^{\circ} 12^{\prime} 01^{\prime \prime}$ de longitude oeste, com uma altitude de 46 metros. O solo é classificado como Argissolo Vermelho-Amarelo, com relevo plano a suavemente ondulado, caracterizado pela Unidade de Paisagem dos tabuleiros costeiros. A região tem clima, de acordo com a classificação de Köeppen, do tipo As. tropical chuvoso, temperatura média anual de $25,2^{\circ} \mathrm{C}$, com verão seco e precipitação pluviométrica média anual de $1.300 \mathrm{~mm}$, com chuvas concentradas nos meses de abril a setembro. Antes da instalação do experimento, foi coletada uma amostra de solo na profundidade de $0-0,20 \mathrm{~m}$, cujos resultados são os seguintes: $\mathrm{pH}\left(\mathrm{H}_{2} \mathrm{O}\right)$ - 5,2; matéria orgânica (M.O.) - $21 \mathrm{~g} \mathrm{dm}^{-3}, \mathrm{~K}-0,8 \mathrm{mmol}_{\mathrm{c}} \mathrm{dm}^{-3}, \mathrm{Al}^{3+}$ - $2 \mathrm{mmol}_{\mathrm{c}} \mathrm{dm}^{-3} \mathrm{e}$ CTC - 44,5 $\mathrm{mmol}_{\mathrm{c}} \mathrm{dm}^{-3}$. Essas análises foram realizadas no Laboratório de Fertilidade do Solo da Embrapa Tabuleiros Costeiros, o qual utiliza volume de solo para as determinações.

O delineamento foi em blocos ao acaso, com quatro repetições, utilizando-se de um esquema fatorial $4^{2}$ para os fatores $\mathrm{N}(0 ; 117 ; 235$ e $352 \mathrm{em} \mathrm{kg}$ $\mathrm{ha}^{-1}$ de $\left.\mathrm{N}\right)$, na forma de ureia, e K $(0 ; 157 ; 313$ e 470, em $\mathrm{kg} \mathrm{ha}^{-1}$ de $\mathrm{K}_{2} \mathrm{O}$ ), na forma de cloreto de potássio. As áreas total e útil das parcelas foram 100 e 40 m², respectivamente. A cultivar estudada foi a PrataAnã. Durante a condução do experimento, foram realizados desbastes dos "filhotes" mais jovens, mantendo-se apenas duas plantas por cova. O cultivo foi irrigado por sistema de microaspersão, tendo os microaspersores uma vazão de $70 \mathrm{~L} \mathrm{~h}^{-1}$. As lâminas de irrigação foram determinadas por meio do uso do Tanque Classe A e de acordo com as necessidades hídricas da cultura, adotando-se o Kc para cada estádio de desenvolvimento da bananeira (Coelho et al., 1999). O manejo da irrigação foi realizado através do acompanhamento do teor de umidade do solo, utilizando-se de tensiômetros e através dos dados da estação meteorológica existente no local do estudo. As fertirrigações foram realizadas mensalmente, ao longo do ciclo fenológico da cultura.

Foram considerados os seguintes componentes de produção: produção de banana; número de pencas por cacho; número de frutos por cacho, e a massa média de fruto de cada cacho, obtido dividindo-se a massa total das pencas pelo número de frutos. No final do segundo ciclo, foram coletadas amostras de solo por tratamento, nas profundidades de 0-0,20 e 0,20-0,40 m, nas quais foram determinados o $\mathrm{pH}\left(\mathrm{H}_{2} \mathrm{O}\right)$ M.O., K, Ca, Mg e $\mathrm{H}+\mathrm{Al}$ de acordo com Embrapa (1979). Essas análises foram realizadas no Laboratório da Universidade Federal de Campina Grande o qual utiliza massa de solo.

Os dados obtidos foram submetidos à análise de variância e de regressão, admitiu-se a probabilidade de $95 \%$.

\section{RESULTADOS E DISCUSSÃO}

O N promoveu aumento linear, no número de frutos por cacho, conforme a Figura 1. Estima-se em 4,3\% o aumento do número de frutos por cacho, 
por incremento da aplicação de $100 \mathrm{~kg}$ de $\mathrm{N}$ por hectare. Brasil et al. (2000) e Borges et al. (2002) também observaram efeito significativo do $\mathrm{N}$ no número de frutos por cacho. Entretanto, no presente estudo, não houve efeito significativo do $\mathrm{N}$ na produção da bananeira. No final do segundo ciclo, o teor de M.O. do solo, no tratamento sem nitrogênio, foi de 23,5 g kg-1 . Borges et al. (2002), avaliando doses de nitrogênio na bananeira Terra, também não obtiveram efeitos significativos na produção, pois, de acordo com os citados autores, o solo onde foi conduzido o experimento continha $42,7 \mathrm{~g} \mathrm{~kg}^{-1}$ de M.O. e 1,8 $\mathrm{g} \mathrm{kg}^{-1}$ de $\mathrm{N}$ total. Além disto, os autores aplicaram 10 litros de esterco de curral em todos os tratamentos, o qual deve ter provido parte do $\mathrm{N}$ requerido pela bananeira. Brasil et al. (2000) obtiveram resposta linear ao nitrogênio, na variável massa do cacho, no segundo ciclo. O resultado obtido está relacionado ao baixo teor de M.O. (13,8 $\left.\mathrm{g} \mathrm{dm}^{-3}\right)$. Esses resultados indicam que o teor de M.O. influencia na resposta ao $\mathrm{N}$ pela bananeira. Silva et al. (2003) observaram redução da produção de banana no $2^{\circ}$ ciclo com o aumento da dose de N. A aplicação de $\mathrm{N}$ elevou o teor de $\mathrm{Mn}$ na folha para $875 \mathrm{mg} \mathrm{kg}^{-1}$, e os autores associaram a queda de produtividade à toxidez do manganês.

Na Figura 2, é mostrada a relação entre a produção de banana e as doses de $\mathrm{K}$, a qual foi significativa e linear. Brasil et al. (2000) avaliaram a produção da bananeira cultivar Pioneira, no segundo ciclo e obtiveram resposta quadrática para a aplicação de $\mathrm{K}$. As doses de $\mathrm{K}_{2} \mathrm{O}$ utilizadas por Brasil et al. (2000) chegaram a $750 \mathrm{~kg}$ de $\mathrm{K}_{2} \mathrm{O} \mathrm{ha}^{-1}$, enquanto no presente trabalho a maior dose estudada foi de $470 \mathrm{~kg}$ de $\mathrm{K}_{2} \mathrm{O} \mathrm{ha-1}$, o que pode não ter sido suficiente para a inflexão da curva de resposta. Silva et al. (2003) não observaram efeito da aplicação de K sobre a produção de banana no segundo ciclo. Esse resultado está relacionado ao elevado teor de K no solo, o qual era de 5,4 mmol $\mathrm{dm}^{-3}$. Na Figura 3, é mostrada a relação entre doses de $\mathrm{K}$ e número de pencas por cacho, a qual foi significativa $(\mathrm{P}<0,01)$. Estima-se em 2,7\% o aumento no número de pencas por cacho por incremento da aplicação de $100 \mathrm{~kg}$ de $\mathrm{K}_{2} \mathrm{O}$ por hectare. $\mathrm{O}$ número de frutos por cacho foi influenciado significativamente pelas doses de K (Figura 4). Estima-se em 3,9\% o aumento no número de frutos por cacho, por incremento da aplicação de $100 \mathrm{~kg}$ de $\mathrm{K}_{2} \mathrm{O}$ por hectare.

Na Figura 5, é mostrada a superfície de resposta para a massa média de frutos, em função de doses de $\mathrm{N}$ e K, cuja interação foi significativa. $\mathrm{O}$ $\mathrm{N}$ diminuiu a massa média dos frutos nas doses mais baixas de $\mathrm{K}$, o mesmo não ocorrendo nas doses mais altas. Entretanto, o efeito do K na massa média do fruto foi mais marcante que o de N. Maia et al. (2003) observaram efeito do K no aumento do peso dos frutos e, consequentemente, no aumento da produtividade.

$\mathrm{O} \mathrm{N}$ e o $\mathrm{K}$ são fundamentais para o desenvolvimento e produção da bananeira; entretanto, faz-se necessário que haja equilíbrio entre as doses aplicadas dos nutrientes, de acordo com as exigências da cultura. Borges e Silva (1995), avaliando a extração de macronutrientes pela bananeira, obtiveram a seguinte ordem decrescente de absorção $\mathrm{K}>\mathrm{N}$. No presente trabalho, ficou evidenciado que as maiores produtividades foram obtidas com a aplicação de maiores doses de K. O K foi o nutriente de efeito mais expressivo para a produção da bananeira, o que pode ser comprovado pelo fato de que as menores produtividades foram obtidas exatamente nos tratamentos que não receberam adubação potássica.

Foram observados efeitos significativos dos tratamentos no teor de K no solo, o qual foi maior na profundidade de $0-0,20 \mathrm{~m}$, indicando acúmulo do nutriente na citada profundidade. Nas Figuras 6a e $6 b$, são mostradas as relações entre as doses de $\mathrm{K}$ e o teor do nutriente no solo, nas duas profundidades. Na profundidade de $0-0,20 \mathrm{~m}$, a relação foi linear, e na de $0,20-0,40 \mathrm{~m}$, a relação foi quadrática. Os teores de K no solo, nas profundidades de 0-0,20 m e 0,20-0,40 $\mathrm{m}$, foram relacionados com a produção de banana (Figuras 7 e 8). Na profundidade de 0-0,20 m, não foi possível estabelecer um nível crítico, pois a relação foi linear (Figura 7). Com base no modelo linear, estima-se em $51 \%$ o aumento da produção de banana por incremento unitário de $\mathrm{K}$ no solo, em mmol $\mathrm{kg}^{-1}$. Na profundidade de 0,20-0,40 m (Figura 8), o nível crítico de $\mathrm{K}$ no solo foi $0,71 \mathrm{mmol}_{\mathrm{c}} \mathrm{kg}^{-1}$ quando analisado pelo método Mehlich-1. 


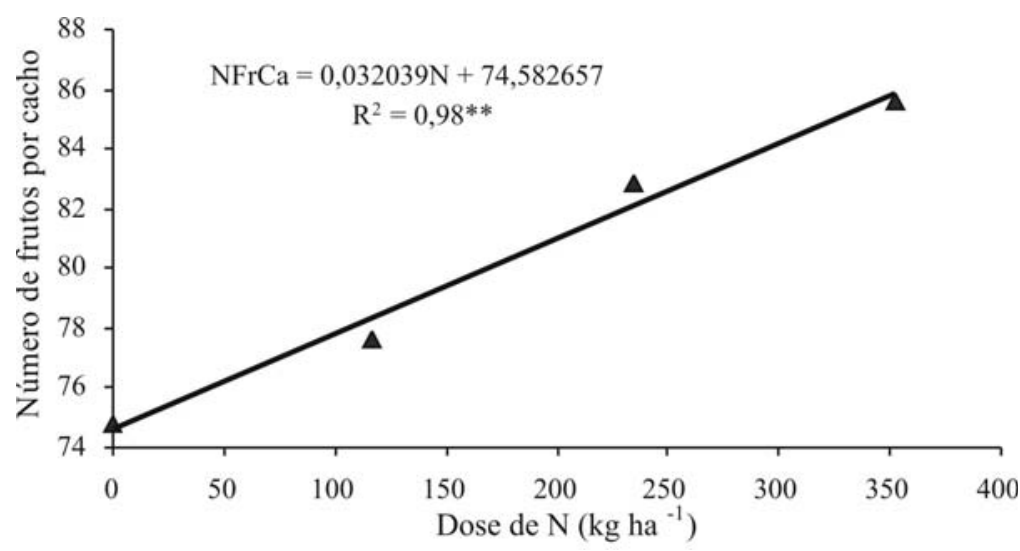

FIGURA 1- Efeitos dos níveis de N no número de frutos por cacho.

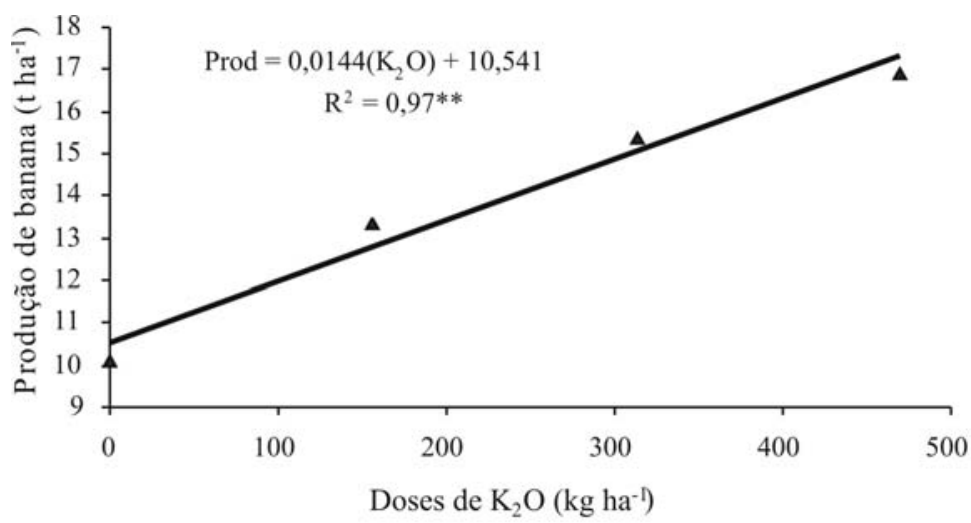

FIGURA 2- Efeitos dos níveis de K na produção de banana.

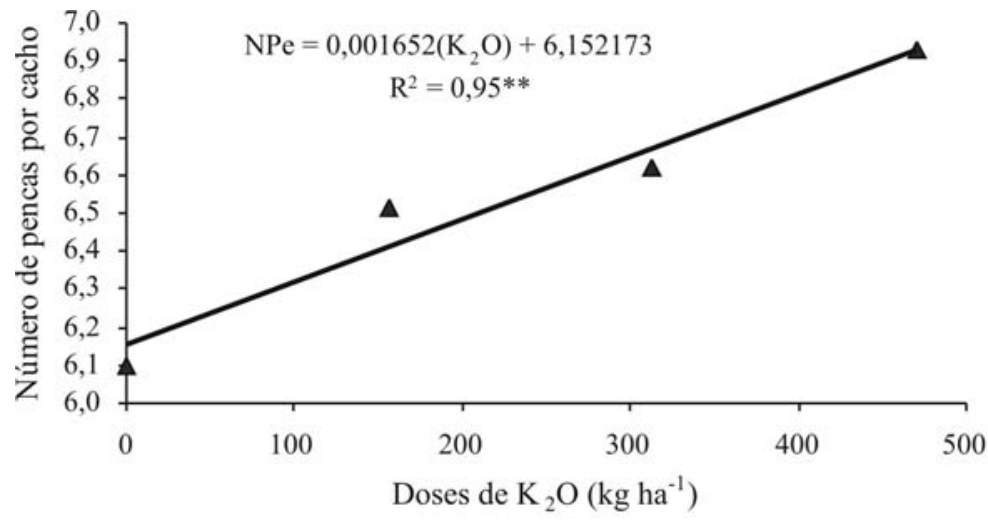

FIGURA 3- Efeitos dos níveis de K no número de pencas por cacho. 


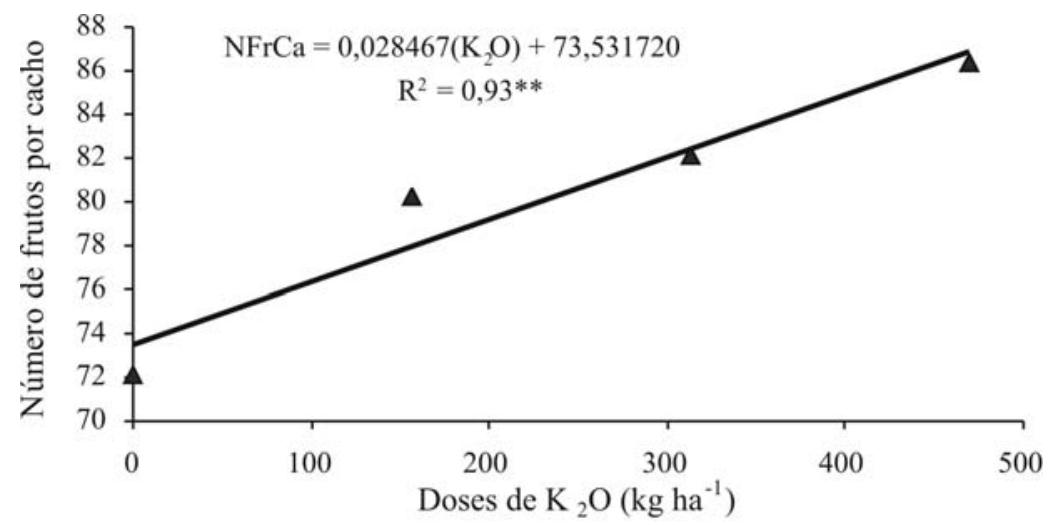

FIGURA 4- Efeitos dos níveis de K no número de frutos por cacho.

$$
\begin{gathered}
\mathrm{Y}=57,7473-0,0332316 \mathrm{~N}+0,119105 \mathrm{~K}_{2} \mathrm{O}-0,1157 \mathrm{e}-3 \mathrm{~K}_{2} \mathrm{O}^{2}+0,68 \mathrm{e}-4 \mathrm{NK}_{2} \mathrm{O} \\
\mathrm{R}^{2}=0,76^{* *}
\end{gathered}
$$

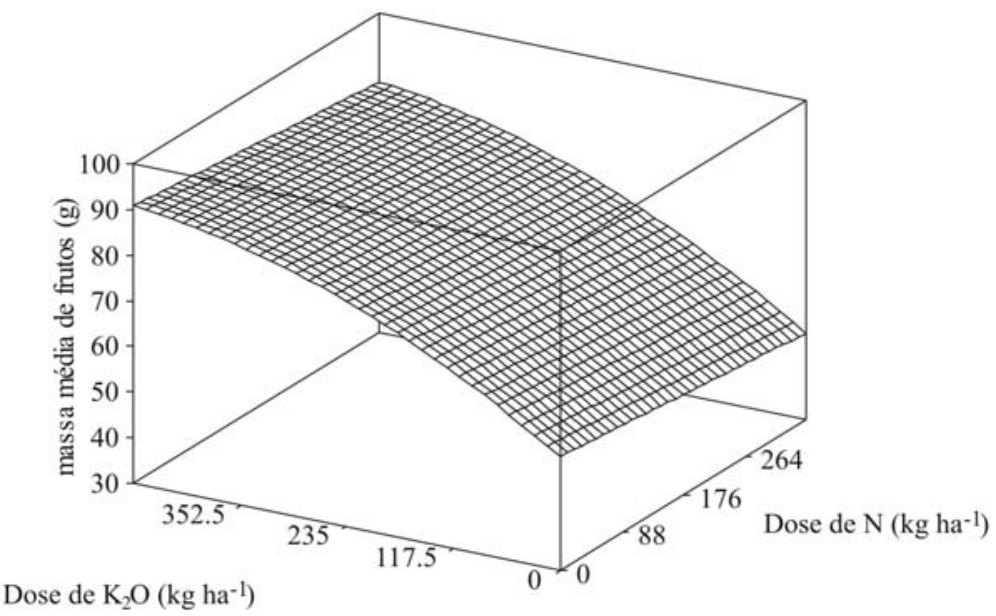

FIGURA 5- Superfície de resposta para a massa média de frutos, em função de doses de N e K.

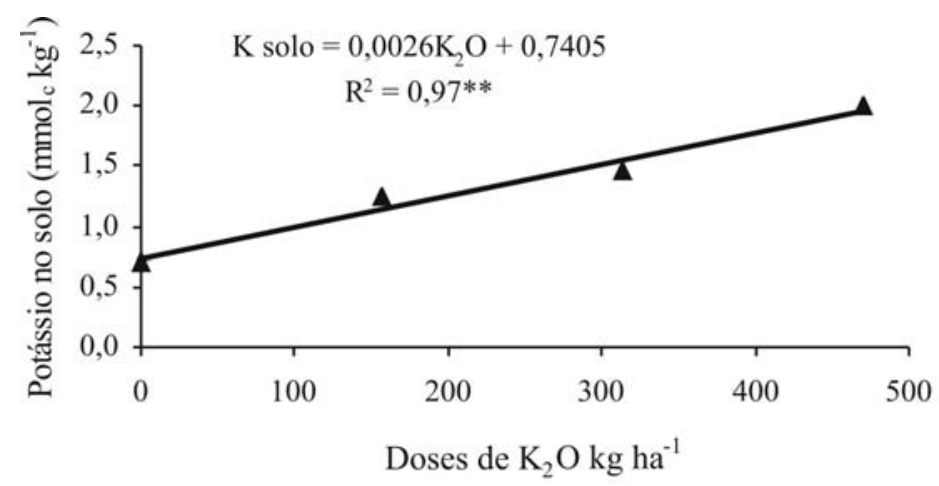

FIGURA 6a- Teor de potássio no solo, na profundidade de 0-0,20 m, em função dos níveis de K. 


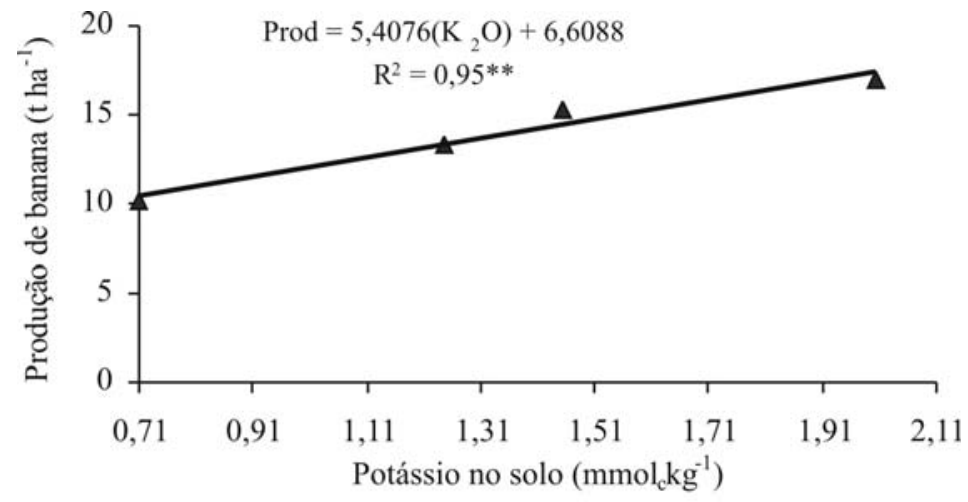

FIGURA 6b- Teor de potássio no solo, na profundidade de 0,20-0,40 m, em função dos níveis de K.

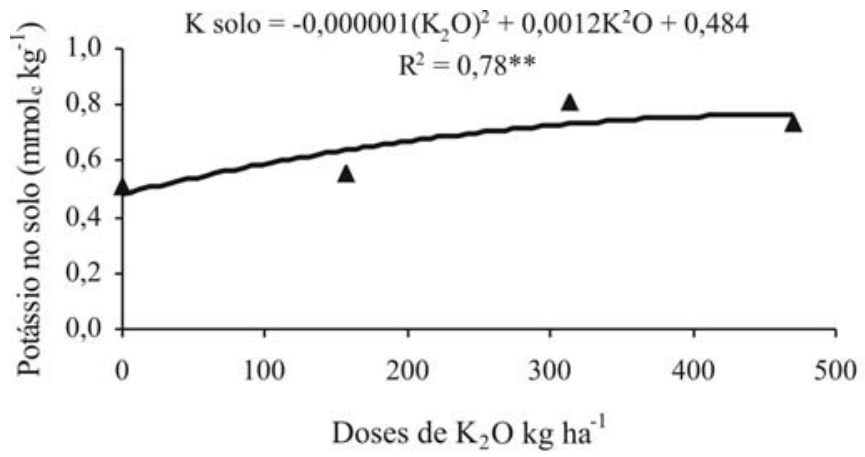

FIGURA 7- Efeito da acumulação do K no solo, na produção de banana, na profundidade de 0-0,20 m.

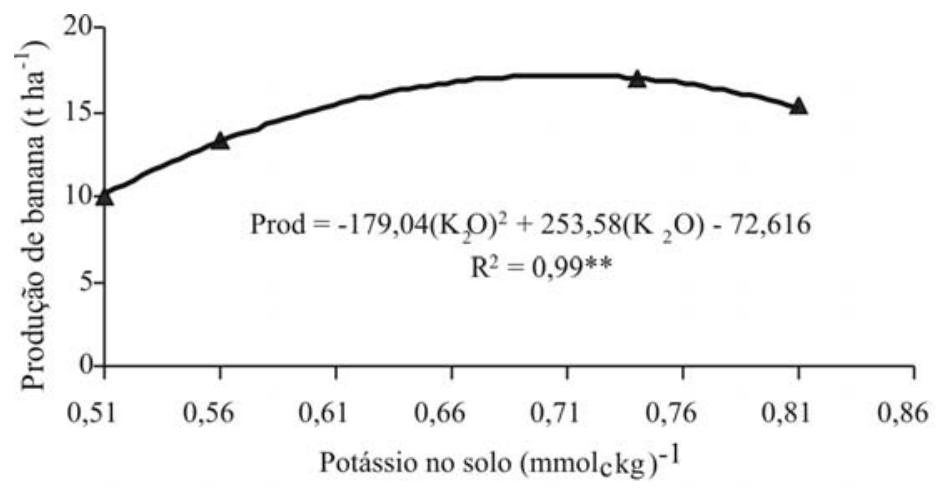

FIGURA 8- Efeito da acumulação do K no solo, na produção de banana, na profundidade de 0,20-0,40 m.

\section{CONCLUSÕES}

1-O N não influenciou na produção de banana, enquanto o K aumentou linearmente a produção.

2-O nível crítico de K pelo Mehlich-1, na profundidade de $0,20-0,40 \mathrm{~m}$, foi $0,71 \mathrm{mmol}_{\mathrm{c}} \mathrm{kg}^{-1}$.

\section{AGRADECIMENTOS}

Ao FINEP pelo financiamento do projeto de pesquisa. A Embrapa Tabuleiros Costeiros e a Universidade Federal de Campina Grande pelas análises de solo. 


\section{REFERÊNCIAS}

BORGES, A.L.; OLIVEIRA, A.M.G. Nutrição, calagem e adubação. In: CORDEIRO, Z.J.M. Banana produção: aspectos técnicos. Frutas do Brasil. Brasília: Embrapa, 2000.p.47-59.

BORGES, A.L.; SILVA, S.O. Extração de macronutrientes por cultivares de banana. Revista Brasileira de Fruticultura, Jaboticabal, v.17, n.1, p.57-66,1995.

BORGES, A.L.; SILVA, T.O.; CALDAS, R.C.; ALMEIDA, I.E. Adubação nitrogenada para bananeira - Terra (Musa sp. AAB, subgrupo Terra). Revista Brasileira de Fruticultura, Jaboticabal, v.24, n.1, p.189-193, 2002.

BRASIL, E.C.; OEIRAS, A.H.L.; MENEZES, A.J.E.A de; VELOSO, C.A.C. Desenvolvimento e produção de frutos de bananeira em resposta à adubação nitrogenada e potássica. Pesquisa Agropecuária Brasileira, Brasília, v.35, p.2407-2414, 2000.

COELHO, E.F.; OR, D.; SOUZA, V.F. Avaliação de parâmetros hidráulicos para modelos de distribuição de água no solo sob gotejamento. Pesquisa Agropecuária Brasileira, Brasília, v.34, n.4, p.651657, 1999.

DUENHAS, L.H.; VILLAS BÔAS, R.L.; SOUZA, C.M.P.; RAGOSO, C.R.A.; BULL, L.T. Fertirrigação com diferentes doses de N P K e seus efeitos sobre a produção e qualidade de frutos de laranja (Citrus sinensis O.) 'Valência'. Revista Brasileira de Fruticultura, Jaboticabal, v. 24, n.1, p. 214-218, 2002.

EMBRAPA. Serviço Nacional de Levantamento e Conservação do Solo. Manual de métodos de análises do solo. Rio de Janeiro, 1979.
GUERRA, A.G; ZANINI, J.R.; NATALE, W.; PAVANI, L.C. Frequência da fertirrigação da bananeira-prataanã com nitrogênio e potássio aplicados por microaspersão. Engenharia Agrícola, Jaboticabal, v.24, n.1, p.80-88, 2004.

LAHAV, E.; TURNER, D. Banana nutricion. Bern, Switzerland: International Potash Institute, 1983. 62p. (Bulletin, 7).

MAIA, V.C.; SAOMÃO, L.C.C.; CANTARUTTI, R.B.; VENEGAS, V.H.A.; COUTO, F.A.D. Efeitos de doses de nitrogênio, fósforo e potássio sobre os componentes da produção e a qualidade de bananas "Prata-Anã” no distrito agroindustrial de Jaíba. Revista Brasileira de Fruticultura, Jaboticabal, v.25, n.2, p.319-322, 2003.

SILVA, T.O.; BORGES, A.L.; CARVALHO, J.G.; DAMASCENO, J.E.A. Adubação com potássio e nitrogênio em três ciclos de produção da bananeira cv. Prata-Anã. Revista Brasileira de Fruticultura, Jaboticabal, v. 25, n. 1, p. 152-155, 2003.

SOUSA, V.F.de; VELOSO, M.E.C.; VASCONCELOS, L.F.L.; RIBEIRO,V.Q.; SOUZA, V.A.B.de; ALBURQUERQUE JÚNIOR, B.S. Nitrogênio e potássio via água de irrigação nas características de produção da bananeira Grand Naine. Pesquisa Agropecuária Brasileira, Brasília, v.39, n.9, p.865869, 2004.

TEIXEIRA, L.A.J.; NATALE, W.; RUGGIERO, C. Alterações em alguns atributos químicos do solo decorrentes da irrigação e adubação nitrogenada e potássica em bananeira após dois ciclos de cultivo. Revista Brasileira de Fruticultura, Jaboticabal, v.23, n.3, p.684-689, 2001.

WARNER, R. M.; FOX, R.L. Nitrogen and potassium nutricion of the Giant Cavendish banana in Hawaii. Journal American Society Horticultural Science, Alexandria, v.102, p. 739-743, 1977. 